\title{
A prospective clinical study of detailed neurological manifestations in patients with COVID-19
}

\author{
Ömer Karadaş ${ }^{1}\left[\right.$ (D) Bilgin Öztürk ${ }^{1}\left(\mathbb{D} \cdot\right.$ Ali Rıza Sonkaya $^{1}$ (B)
}

Received: 1 June 2020 / Accepted: 21 June 2020 / Published online: 25 June 2020

(C) Fondazione Società Italiana di Neurologia 2020

\begin{abstract}
Background COVID-19 is a virus pandemic. According to the first obtained data, COVID-19 has defined with findings such as cough, fever, diarrhea, and fatigue although neurological symptoms of patients with COVID-19 have not been investigated in detail. This study aims to investigate the neurological findings via obtained face-to-face anamnesis and detailed neurological examination in patients with COVID-19.

Methods Two hundred thirty-nine consecutive inpatients with COVID-19, supported with laboratory tests, were evaluated. Detailed neurological examinations and evaluations of all patients were performed. All evaluations and examinations were performed by two neurologists who have at least five-year experience.

Results This study was carried out 239 patients (133 male +106 female) with diagnosed COVID-19. Neurological findings were present in 83 of 239 patients (34.7\%). The most common neurological finding was a headache (27.6\%). D-dimer blood levels were detected to be significantly higher in patients with at least one neurological symptom than patients without the neurological symptom $(p<0.05)$. IL-6 level was found to be significantly higher in patients with headache than without headache $(p<0.05)$. Creatine kinase $(\mathrm{CK})$ level was detected to be significantly higher in patients with muscle pain $(p<0.05)$.

Conclusion Neurological symptoms are often seen in patients with COVID-19. Headache was the most common seen neurological symptom in this disease. Dizziness, impaired consciousness, smell and gustation impairments, cerebrovascular disorders, epileptic seizures, and myalgia were detected as other findings apart from the headache. It is suggested that determining these neurological symptoms prevents the diagnosis delay and helps to prohibit virus spread.
\end{abstract}

Keywords Coronavirus disease $2019 \cdot$ COVID-19 $\cdot$ SARS-CoV-2 $\cdot$ Neurologic symptoms $\cdot$ Neurological manifestations

\section{Introduction}

It was informed to be pneumonia cases that rapidly increased and completely undetermined etiology in Wuhan, China. A previously unknown virus type belonging to coronavirus family has been described as the etiological reason of these pneumonia cases. In following days, the World Health Organization (WHO) has defined the disease name as a COVID-19, which means 2019 coronavirus disease [1]. Virus that caused to COVID-19 has been named as a SARS-CoV-2 because of the similarity of SARS-CoV which was previously caused the

Bilgin Öztürk

Drbilgin@gmail.com

1 University of Health Science, Gulhane School of Medicine, Neurology Department, Ankara, Turkey similar pandemic (severe acute respiratory syndrome) as well [2-4]. COVID-19 causes the acute respiratory syndrome. It is accepted to be a life-threatening disease because of the serious lower respiratory tract involvement.

COVID-19 infection symptoms arise from after approximately 5 days incubation period [5]. The most common symptoms of COVID-19 are fever, cough, and fatigue. Apart from these, headache, dyspnea, and diarrhea are the other detected findings of disease [6]. Pneumonia, acute respiratory distress syndrome, and multi-organ failure may develop in the most severe cases. Neurotropic and neuroinvasive features of coronavirus have been defined [7]. Coronavirus reaches to the central nerve system (CNS) via nervous olfactory with nasal infection, and then it can cause the inflammation and demyelization [7].

Coronavirus types use similar viral structures and infection routes. Pathological mechanisms which were found for other known coronaviruses subtypes can also be considered for 
COVID-19 [8]. Coronaviruses do not always affect only human respiratory system. It can affect the CNS [8]. According to the obtained data, neurological involvement is not rare in human coronaviruses infections. Virus was detected from cerebrospinal fluid (CSF) in reported SARS-CoV patients [9]. In one study conducted on 70 patients with MERS-CoV infection, it was reported an epileptic seizures and mental state changes [10]. Therefore, it is possible to detect the neurological symptoms in COVID-19 patients with detailed neurological examination.

Coronaviruses may be found in many animals and may cause systemic infection. Coronavirus types such as SARS$\mathrm{CoV}$ and Middle East respiratory syndrome CoV (MERS$\mathrm{CoV}$ ) cause the pandemic [2-4, 6]. Also, COVID-19 infection causes the serious clinic symptoms and high mortality in humans. In the literature, clinical symptoms such as fever, cough, dyspnea, diarrhea, and fatigue have often been described in studies regarding with COVID-19, but data related to neurological findings are not enough $[8,11]$. Therefore, to fill this gap in the literature, in this study, 239 patients with COVID-19 confirmed with laboratory tests were evaluated in detail from a neurological point of view. Neurological involvements obtained with this evaluation were reported.

\section{Materials and methods}

In this prospective study, 239 consecutive inpatients, diagnosed with COVID-19 according to WHO interim guidance, were investigated from April 2020 to May 2020. COVID-19 positivity has been defined with the real-time reverse transcription polymerase chain reaction (rRT-PCR) analysis of the throat and nasal swab specimens which is using for SARS-CoV-2 virus analysis. Thorax CT and laboratory tests such as a complete blood cell count, blood analysis, coagulation testing, D-dimer, assessment of liver and renal function testing, C-reactive protein, creatinine kinase (CK), and lactate dehydrogenase (LDH) were investigated as standard in all patients. Fever was described if body temperature is $\geq 37^{\circ} \mathrm{C}$.

Patients who are unable to communicate were excluded from the study. Two hundred seventy-nine hospitalized patients with laboratory confirmation of COVID-19 were included in the study. This prospective clinical study was approved by the Local Ethic Committee (Protocol No: 2020162). Before the study, informed consent form was taken from all patients. The study was performed in accordance with the principles of Helsinki Declaration.

\section{Data collection}

Demographic features (age, gender), fever, and neurological symptoms were recorded in patients with COVID-19.
Neurological examination and evaluation were performed with personal protective equipment in all patients. Brain CT/ MRI and/or electroencephalography (EEG) investigations were performed according to the neurological symptoms in need. High-resolution thorax CT (HRCT) and laboratory findings (IL-6, D-dimer, and CK) of all patients were evaluated.

Neurological examination and evaluation were performed and confirmed by two trained neurologists working in COVID clinic. All patients were conscious, coopered, and oriented. All neurological findings were obtained via face-to-face.

\section{Statistical analysis}

The collected data were assessed with SPSS for Windows 26. To summarize the features of demographic data, descriptive statics were used. Qualitative variables were indicated with frequency and percentage; numerical variables were summarized by mean \pm standard deviation. To compare the variables, $t$ tests were performed, and $p<0.05$ was accepted as statistically significant.

\section{Results}

This prospective study was conducted on hospitalized patients diagnosed with COVID-19, with neurological examination and face-to-face interview between April and May 2020. Two hundred thirty-nine patients (133 males + 106 females) with diagnosed COVID-19 were included in the study. The mean age of the patients was $46.46 \pm 15.41(\min 19$, $\max 88)$. The demographic characteristics of patients are shown in the Table 1.

Neurological findings were observed in 83 patients (34.7\%). Fever was the most common symptom in patients with COVID-19 (33.1\%). The most common neurological finding was headache (27.6\%). The characteristic of headache was detected usually bilateral; it was mostly seen on frontal and posterior areas of the head.

IL-6 results were obtained in 28 of 239 patients. Thirteen patients had complaint of headache, and 15 patients had no complaint of headache among these patients. The mean value

Table 1 Demographic characteristics of patients

\begin{tabular}{lllll}
\hline Characteristics & $\begin{array}{l}\text { All patients } \\
(n=239)\end{array}$ & Minimum & Maximum & Mean \pm SD \\
\hline $\begin{array}{l}\text { Age, years } \\
\text { Sex }\end{array}$ & & 19.00 & 88.00 & $46.46 \pm 15.41$ \\
Female & $106(44.4 \%)$ & & \\
Male & $133(55.6 \%)$ & & \\
\hline
\end{tabular}


of IL-6 was $57.18 \pm 52.95 \mathrm{pg} / \mathrm{mL}$ (min $17.32 \mathrm{pg} / \mathrm{mL}$, $\max$ $214.3 \mathrm{pg} / \mathrm{mL}$ ) in patients with complaint of headache. The mean value of IL-6 was $42.94 \pm 28.95 \mathrm{pg} / \mathrm{mL}$ (min $2.70 \mathrm{pg}$ / $\mathrm{mL}$, $\max 114.13 \mathrm{pg} / \mathrm{mL}$ ) in patients with no complaint of headache. IL-6 level was found to be significantly higher in patients with headache $(p<0.05)$.

Besides the headache, trigeminal neuralgia was detected in 8 patients $(3.3 \%)$, and glossopharyngeal neuralgia was found in 9 patients $(3.7 \%)$.

There was a small impairment in 18 patients $(7.5 \%)$ and a taste impairment in 16 patients $(6.7 \%)$. Of the 16 patients with taste impairments, 11 had also smell impairments.

Cerebrovascular disease (CVD) was found in 9 patients $(3.8 \%)$. Two of these patients were evaluated as hemorrhagic CVD, 3 as transient ischemic attack (TIA), and 4 as ischemic CVD. Muscle pain was present in $36(15.1 \%)$ patients, and it was one of the most common neurological findings. CK results were obtained in 140 of the 239 patients. Thirty six of 140 patients had a muscle pain and 104 patients had no muscle pain complaint. The mean CK values were $241.05 \pm$ $137.02 \mathrm{U} / \mathrm{L}(\min 45.00 \mathrm{U} / \mathrm{L}, \max 721 \mathrm{U} / \mathrm{L})$ and $139.67 \pm$ $83.80 \mathrm{U} / \mathrm{L}(\min 21 \mathrm{U} / \mathrm{L}, \max 451 \mathrm{U} / \mathrm{L}$ ), respectively. CK level was found to be significantly higher in patients with muscle pain $(p<0.05)$.

D-dimer blood levels were compared between patients having at least one neurological symptom $(n=83)$ and without any neurological symptoms $(n=156)$. The mean value of $\mathrm{D}$ dimer blood levels was $2.38 \pm 4.74 \mathrm{mg} / \mathrm{L}(\min 0.19 \mathrm{mg} / \mathrm{L}$, $\max 42.3 \mathrm{mg} / \mathrm{L})$ and $0.53 \pm 0.45 \mathrm{mg} / \mathrm{L}(\min 0.1 \mathrm{mg} / \mathrm{L}, \max$ $2.25 \mathrm{mg} / \mathrm{L}$ ), respectively. D-dimer level was detected to be significantly higher in patients with neurological symptoms $(p<0.05)$.

Sleep complaints were present in 30 patients $(12.6 \%)$. Twenty one of these 30 patients had at least one of the symptoms such as headache, trigeminal neuralgia, and glossopharyngeal neuralgia.

Four patients $(1.7 \%)$ had complaints which overlap with the restless leg syndrome (RLS) criteria when it was questioned to them. These patients stated that they did not have such a diagnosis before hospitalization and that they did not have such significant complaints. Neurological findings are summarized in Table 2. One patient was diagnosed as Guillain-Barre Syndrome (GBS) 2 weeks after discharging with COVID-19 and rehospitalized.

\section{Discussion}

In this prospective study, neurological symptoms in hospitalized patients with COVID-19 were evaluated. Eighty three $(34.7 \%)$ of 239 patients were evaluated with neurological involvement. There were several neurological symptoms including CNS, PNS, and skeletal muscles. The most common typical
Table 2 Neurological findings

\begin{tabular}{ll}
\hline Sign and Symptoms & All patients $(N=239)$ \\
\hline Fever & $79(33.1 \%)$ \\
Headache & $64(26.7 \%)$ \\
Trigeminal neuralgia & $8(3.3 \%)$ \\
Glossopharyngeal neuralgia & $9(3.7 \%)$ \\
Vasoglossopharyngeal neuralgia & $2(0.8 \%)$ \\
Pain with eye movements & $3(1.3 \%)$ \\
Dizziness & $16(6.7 \%)$ \\
Tinnitus & $5(2.1 \%)$ \\
Lack of hearing & $3(1.3 \%)$ \\
Visual defect & $8(3.3 \%)$ \\
Numbness on tongue & $4(1.7)$ \\
Bifurcation in voice & $3(1.3 \%)$ \\
Numbness in the face area & $8(3.3 \%)$ \\
Smell impairment & $18(7.5 \%)$ \\
Taste impairment & $16(6.7 \%)$ \\
Cerebrovascular disorders & $9(3.8 \%)$ \\
Hemorrhagic CVD & $2(0.8 \%$ \\
Ischemic CVD & $7(2.9 \%)$ \\
Impaired consciousness-confusion & $23(9.6 \%)$ \\
Sleep disorder & $30(12.6 \%)$ \\
Orthostatic hypertension & $8(3.3 \%)$ \\
Balance disorder & $6(2.5 \%)$ \\
Muscle pain & $36(15.1)$ \\
Guillain-Barre syndrome & $1(0.4 \%)$ \\
Restless leg syndrome & $4(1.7 \%)$ \\
Nausea & $13(5.43 \%)$ \\
Diarrhea & $13(5.4 \%)$ \\
\hline & \\
&
\end{tabular}

symptom was fever $(33.1 \%)$. Headache was the most detected symptom among the neurological symptoms (26.7\%). Neurological manifestation must be paid attention in patients with COVID-19. Neurological symptoms should be considered as an important finding during the COVID-19 pandemic.

Although respiratory symptoms of COVID-19 are at the forefront, it has been understood that COVID-19 causes the important neurological symptoms [8, 11]. In addition, this virus has also been detected in CSF, and it is understood that it will potentially cause the nerve system damage [9]. Pathological mechanism of COVID-19 is similar with SARS and MERS viruses. Similar to other corona viruses such as SARS and MERS, SARS-CoV-2 can affect the nerve systems either hematogenous or retrograde neuronal route. On the other hand, it has been thought to be that COVID-2019 may disrupt the blood-brain barrier [7]. This study is a manifestation of nervous system symptoms in COVID-19 patients and an indicative of neurological involvement. Some autopsy results of patients with coronaviruses confirmed the neurological damage $[12,13]$. 
Studies have remarked that cytokines such as IL-6 and TNF- $\alpha$ are pain mediators in neurovascular inflammation [14]. It has been reported that cytokines such as IL-6 are particularly painful mediators in migraine, and IL-6 levels are significantly higher in some headache patients [15]. IL-6 is a proinflammatory cytokine released from $\mathrm{T}$ cells and macrophages as the stimulant of the immune response. It is known to cause inflammation in cases such as infection and trauma. In addition, this cytokine, which is an important mediator of fever and acute phase response and initiates PGE2 synthesis in the hypothalamus by easily crossing the blood-brain barrier, has been found to be produced by smooth muscle cells of many blood vessels [16].

Angiotensin-converting enzyme 2 (ACE2) is present in multiple human organs such as nervous system and skeletal muscles [17]. ACE2 plays role in regulating the blood pressure. The blood-brain barrier may be disrupted during COVID-19 via endothelium dysfunctions [12]. Thereby, SARS-CoV-2 may cause some neurologic manifestations via direct or indirect mechanisms. In this study, it was found that CK levels in patients with skeletal muscle injury were higher than those without muscle symptoms. SARS-CoV-2 may affect the skeletal muscle cells alone or with proinflammatory cytokines by binding with ACE2. D-dimer blood levels were higher in patients with neurological symptoms than patients without neurological symptoms. Several researches described the association between D-dimer levels and COVID-19 severity $[8,11]$. Some authors also declared that neurological symptoms are more often in severe COVID-19 cases [11].

The most common neurological symptom in our study was a headache. Skeletal muscle pain, sleep disturbance, and consciousness disorders were also common neurological symptoms. Coronavirus causes the alveolar inflammation and edema [13]. This condition may cause hypoxia in CNS, and this can affect the cerebral blood flow and intracranial pressure resulting with neurological symptoms ranging from headache to coma.

This study has several limitations. Firstly, it could have performed multi-centered and with more patients. Secondly, neuroimaging techniques (brain CT, brain MRI, fMRI, DTI, transcranial Doppler), lumbar puncture, neuroelectrophysiological tests (EEG, electroneuromyography, evoked potentials), and blood analyzes were either not performed or were limited in the epidemic period of COVID-19. And also, we were insufficient to distinguish whether these neurologic manifestations are caused by the virus directly or another organ damage indirectly.

\section{Results}

As a result, COVID-19 affects the nerve and muscle system. The most common neurological symptoms are headache, muscle pain, sleep disorder, impaired consciousness, smell and taste impairments, dizziness, and cerebrovascular diseases. In particular, the headache character was different from other primary headaches. Headaches were more focal and more localized in the frontal and occipital areas. In this study, it was found that neurological findings have an important role in the COVID-19. Being aware of this knowledge may help us to learn the neurological aspects of COVID-19 pandemic. In the future, studies explaining the underlying pathophysiological mechanisms of neurological symptoms in COVID-19 patients are needed.

\section{Compliance with ethical standards}

Conflict of interest The authors declare that they have no conflict of interest.

Ethical approval This prospective clinical study was approved by the Local Ethic Committee (Protocol No: 2020162). Before the study, informed consent form was taken from all patients. The study was performed in accordance with the principles of Helsinki Declaration.

\section{References}

1. https://www.who.int/dg/speeches/detail/who-director-general-sremarks-at-the-media-briefing-on-2019-ncov-on-11-february2020. Accessed.24.05.2020

2. Ksiazek TG, Erdman D, Goldsmith CS, Zaki SR, Peret T, Emery S, Tong S, Urbani C, Comer JA, Lim W, Rollin PE, Dowell SF, Ling AE, Humphrey CD, Shieh WJ, Guarner J, Paddock CD, Rota P, Fields B, DeRisi J, Yang JY, Cox N, Hughes JM, LeDuc J, Bellini WJ, Anderson LJ, SARS Working Group (2003) A novel coronavirus associated with severe acute respiratory syndrome. N Engl J Med 348(20):1953-1966

3. Drosten C, Günther S, Preiser W, van der Werf S, Brodt HR, Becker S, Rabenau H, Panning M, Kolesnikova L, Fouchier RAM, Berger A, Burguière AM, Cinatl J, Eickmann M, Escriou N, Grywna K, Kramme S, Manuguerra JC, Müller S, Rickerts V, Stürmer M, Vieth S, Klenk HD, Osterhaus ADME, Schmitz H, Doerr HW (2003) Identification of a novel coronavirus in patients with severe acute respiratory syndrome. N Engl J Med 348(20): 1967-1976

4. de Groot RJ, Baker SC, Baric RS, Brown CS, Drosten C, Enjuanes L, Fouchier RAM, Galiano M, Gorbalenya AE, Memish ZA, Perlman S, Poon LLM, Snijder EJ, Stephens GM, Woo PCY, Zaki AM, Zambon M, Ziebuhr J (2013) Commentary: Middle East respiratory syndrome coronavirus (MERS-CoV): announcement of the coronavirus study group. J Virol 87(14):7790-7792

5. Lauer SA, Grantz KH, Bi Q, Jones FK, Zheng Q, Meredith HR, Azman AS, Reich NG, Lessler J (2020) The incubation period of coronavirus disease 2019 (COVID-19) from publicly reported confirmed cases: estimation and application. Ann Intern Med 172:577-582

6. Kuiken T, Fouchier RAM, Schutten M, Rimmelzwaan GF, van Amerongen G, van Riel D, Laman JD, de Jong T, van Doornum G, Lim W, Ling AE, Chan PKS, Tam JS, Zambon MC, Gopal R, Drosten C, van der Werf S, Escriou N, Manuguerra JC, Stöhr K, Peiris JSM, Osterhaus ADME (2003) Newly discovered coronavirus as the primary cause of severe acute respiratory syndrome. Lancet 362(9380):263-270

7. Li YC, Bai WZ, Hashikawa T (2020) The neuroinvasive potential of SARS-CoV2 may play a role in the respiratory failure of COVID-19 patients. J Med Virol 
8. Mao L, et al (2020) Neurological manifestations of hospitalized patients with COVID-19 in Wuhan, China: a retrospective case series study

9. Asadi-Pooya AA, Simani L (2020) Central nervous system manifestations of COVID-19: A systematic review. J Neurol Sci 413: 116832

10. Saad M, Omrani AS, Baig K, Bahloul A, Elzein F, Matin MA, Selim MAA, Mutairi MA, Nakhli DA, Aidaroos AYA, Sherbeeni NA, al-Khashan HI, Memish ZA, Albarrak AM (2014) Clinical aspects and outcomes of 70 patients with Middle East respiratory syndrome coronavirus infection: a single-center experience in Saudi Arabia. Int J Infect Dis 29:301-306

11. Huang C, Wang Y, Li X, Ren L, Zhao J, Hu Y, Zhang L, Fan G, Xu J, Gu X, Cheng Z, Yu T, Xia J, Wei Y, Wu W, Xie X, Yin W, Li H, Liu M, Xiao Y, Gao H, Guo L, Xie J, Wang G, Jiang R, Gao Z, Jin Q, Wang J, Cao B (2020) Clinical features of patients infected with 2019 novel coronavirus in Wuhan, China. Lancet 395(10223):497506

12. Varga Z, Flammer AJ, Steiger P, Haberecker M, Andermatt R, Zinkernagel AS, Mehra MR, Schuepbach RA, Ruschitzka F, Moch H (2020) Endothelial cell infection and endotheliitis in COVID-19. Lancet 395(10234):1417-1418
13. Xu Z, Shi L, Wang Y, Zhang J, Huang L, Zhang C, Liu S, Zhao P, Liu H, Zhu L, Tai Y, Bai C, Gao T, Song J, Xia P, Dong J, Zhao J, Wang FS (2020) Pathological findings of COVID-19 associated with acute respiratory distress syndrome. Lancet Respir Med 8(4): 420-422

14. Moore JB, June CH (2020) Cytokine release syndrome in severe COVID-19. Science 368(6490):473-474

15. Karadaş Ö, Özön AÖ, Özçelik F, Özge A (2017) Greater occipital nerve block in the treatment of triptan-overuse headache: a randomized comparative study. Acta Neurol Scand 135(4):426-433

16. Zhang $\mathrm{C}$ et al (2020) The cytokine release syndrome (CRS) of severe COVID-19 and Interleukin-6 receptor (IL-6R) antagonist tocilizumab may be the key to reduce the mortality. Int $\mathrm{J}$ Antimicrob Agents 55:105954

17. Hamming I, Timens W, Bulthuis MLC, Lely AT, Navis GJ, van Goor H (2004) Tissue distribution of ACE2 protein, the functional receptor for SARS coronavirus. a first step in understanding SARS pathogenesis. J Pathol 203(2):631-637

Publisher's note Springer Nature remains neutral with regard to jurisdictional claims in published maps and institutional affiliations. 